\title{
Expression of miR-146a and miR-155 in Egyptian patients with Behçet's disease: clinical significance and relationship with disease activity
}

\author{
Engy El Khateeb', Ayatallah Nassef ${ }^{1}$, Rasha Gheith², Aya Erfan ${ }^{1}$ and Walaa Abdelfattah ${ }^{1 *}$ (D)
}

\begin{abstract}
Background: Behçet's disease (BD) is a systemic vasculitis disorder with multifactorial immunopathogenesis and associated with significant morbidity and mortality. MicroRNAs (miRNAs) are involved in the pathogenesis of inflammatory diseases. MiR-146 and miR-155 are known key regulators of immune response. This study was conducted to determine the expression of miRNA-146a and miRNA-155 in patients with BD and to link their possible association with the clinical manifestations and activity of this disease to evaluate their role as diagnostic or prognostic markers. A total of 60 patients with BD and 25 age- and gender-matched healthy controls were examined in a case-control study from October 2017 to September 2018 for the expression levels of miR-146a and miR-155 using singleplexTaqMan two-step stem loop quantitative reverse transcription real-time polymerase chain reaction (qRT-PCR).
\end{abstract}

Results: Patients with BD had significantly lower miR-146a levels than control subjects $(P<0.001)$. Regarding the miR-155 expression level, no statistically significant differences were detected between patients and healthy controls $(P=0.736)$. The expression level of miR-146a showed no significant association with the different clinical manifestations of patients with BD.

Conclusion: This study suggests the possibility that miR-146a expression in patients with BD is involved in the pathogenesis of disease. Furthermore, it can be used as a diagnostic biomarker and a therapeutic target for BD in the future.

Keywords: Behçet's disease, Behçet's Disease Current Activity Form, MicroRNA-146a, MicroRNA-155, Quantitative reverse transcription real-time polymerase chain reaction

\section{Background}

Behçet's disease (BD) is a chronic, relapsing, inflammatory disease affecting almost all organ systems with characteristic exacerbations and remissions [1] and significant organthreatening morbidity and mortality [2] that may result from widespread inflammatory effects on both arteries and veins of all sizes. Egypt is known to have a high

\footnotetext{
*Correspondence: walaafattah@cu.edu.eg

${ }^{1}$ Clinical and Chemical Pathology Department, Faculty of Medicine, Cairo University, Cairo, Egypt

Full list of author information is available at the end of the article
}

incidence of BD with a higher male-to-female ratio and a higher incidence of neurological (34.9\%) and vascular (57.1\%) lesions than other countries [3].

The pathogenesis of BD might involve genetic susceptibility, environmental factors, as well as inflammatory response abnormalities and abnormal immune responses [4]. Typical presentations include the triad of oral ulcers, genital ulcers, and uveitis. Nevertheless, the skin, gastrointestinal tract, kidneys, lungs, heart, blood vessels, and the central nervous system are also commonly affected [5].

\section{Springer Open}

(c) The Author(s). 2020 Open Access This article is licensed under a Creative Commons Attribution 4.0 International License, which permits use, sharing, adaptation, distribution and reproduction in any medium or format, as long as you give appropriate credit to the original author(s) and the source, provide a link to the Creative Commons licence, and indicate if changes were made. The images or other third party material in this article are included in the article's Creative Commons licence, unless indicated otherwise in a credit line to the material. If material is not included in the article's Creative Commons licence and your intended use is not permitted by statutory regulation or exceeds the permitted use, you will need to obtain permission directly from the copyright holder. To view a copy of this licence, visit http://creativecommons.org/licenses/by/4.0/. 
Due to the lack of pathognomonic signs or specific laboratory, radiologic, or histologic findings for BD [6], its diagnosis depends on the presence of a number of clinical findings defined by a set of diagnostic criteria, which are known as the International Criteria of Behçet's Disease (ICBD), which define that seven clinical items with a total score of 4 points is diagnostic of BD [7].

MicroRNAs (miRNAs), one of the noncoding RNA families, play a significant role in mediating the posttranscriptional regulation of gene expression [8] and controlling biological events, including development, proliferation, differentiation, cell fate determination, apoptosis, signal transduction, and organ development $[9,10]$. Dysregulations in miRNAs have also been implicated in a wide range of diseases, including diabetes, cancer, and cardiovascular, renal, and autoimmune disorders [11]. Among the various miRNAs playing a possible role in regulating the immune system, miR-146 (miR-146a and miR-146b) and miR-155 have emerged as key regulators of the immune response [12].

MiR-146 is a multifunctional miRNA that enhances the apoptosis of dendritic cells (DCs), suppresses the production of proinflammatory cytokines such as TNF$\alpha$, suppresses Th1 and Th17 cells, and activates T regulatory lymphocytes (Tregs) [13]. MiR-146 deficiency results in an excessive production of IL6 and TNF- $\alpha$, myeloproliferative diseases, and inflammatory diseases [14]. On the other hand, miR-155 activates DCs and increases the production of inflammatory cytokines; stimulates the differentiation of Th1 and Th17 cells, proliferation of B cells, and antibody production; and is required for maturation and effector functions of natural killer and CD8 cells [12].

BD activity correlates with a proinflammatory shift in peripheral $\mathrm{T}$ cell populations characterized by the expansion of both Th17 and Th22 subsets and a reduction in Tregs. Dysregulation in miRNAs mediating the immune response pathways and the effector cell development may be involved in these population shifts [15]. Regarding BD, there is limited information about the expression of miRNAs, and furthermore, no highthroughput miRNA expression studies have been conducted to identify miRNAs specifically associated with this disease [16].

The objective of this study was to determine the expression levels of miRNA-146a and miRNA-155 in Egyptian patients with $\mathrm{BD}$ and analyze their possible association with various manifestations of this disease.

\section{Methods}

This study was conducted on 85 participants who were divided into two groups. The first group consisted of 60 Egyptian patients with $\mathrm{BD}$ diagnosed according to the ICBD [7]. There were 6 female and 54 male patients aged 23-65 years (mean $36.33 \pm 8.32$ years). These patients were recruited from the Rheumatology Department, Cairo University, during October 2017 to September 2018. The second group, i.e., the control group, consisted of 25 age- and sex-matched healthy subjects, including 6 females and 19 males, with an age range of $21-60$ years (mean $36.2 \pm 10.4$ years).

All measurements were performed according to the ethical standards of Kasr-Alainy Cairo University Hospitals research committee and the ethical standards of the 1964 Declaration of Helsinki. Informed consent was obtained directly from each subject before data collection and after the explanation of the study objectives. Patients with BD suffering from severe infections, malignant tumors, or other autoimmune diseases were excluded.

All patients with BD were subjected to full historytaking and clinical examination, including disease activity assessment using the Behçet's Disease Current Activity Form (BDCAF) [17]. The severity levels of $\mathrm{BD}$ were graded as mild, moderate, and severe [18]. Routine laboratory investigations consisting of ESR, qualitative CRP, complete blood count, liver function tests (ALT and AST), and kidney function tests (creatinine and urea) were performed for all patients and control subjects.

\section{Determination of serum levels of two mature miRNAs (miR-146a and miR-155)}

Peripheral blood samples $(3 \mathrm{~mL})$ were collected in serum vaccutainer tubes and centrifuged at $3000 \mathrm{rpm}$ for 10 min at room temperature. The supernatant was transferred to Eppendorf tubes. These samples were again centrifuged at $15,000 \mathrm{rpm}$ for $10 \mathrm{~min}$ to precipitate the cell debris, and the resulting supernatants were stored at - $80^{\circ} \mathrm{C}$ until RNA extraction. RNA for miRNA expression profiling was isolated from the serum using the Qiagen ${ }^{\circ}$ miRNeasy Serum/Plasma Kit (Catalog no. 217184) according to the manufacturer's instructions. miR-146a and miR-155 were detected using the Taq$\mathrm{Man}^{\circ}$ miRNA assay. U6 snRNA was used as endogenous control for normalization. Sequences of mature miRNAs and endogenous control were identified using the miRbase shown in Table 1.

RNA was reverse-transcribed into cDNA using the $\mathrm{TaqMan}^{\circ}$ MicroRNA RT Kit (Applied Biosystems, USA

Table 1 Target MiRNA and endogenous control sequences to be studied and amplified by RT-qPCR

\begin{tabular}{lll}
\hline MiRNA & MiRbase No. & Mature sequence \\
\hline U6snRNA & NR_004394 & GTGCTCGCTTCGGCAGCACATATACTAAAA \\
& & TGGAACGATACAGAGAGATTAGCATGG \\
& CCCCTGGCAAGGATGACACGCAAATTCG \\
MiR-146a & MIMAT0000449 & UGAGAACUGAAUUCCAUGGGUU \\
MiR-155 & MIMAT0000646 & UUAAUGCUAAUCGUGAUAGGGGU \\
\hline
\end{tabular}


Part No. 4366596), in combination with the stem loopTaqMan MicroRNA and control RT primer $5 \times$ (Applied Biosystems, USA, part No. 4427975), allowing the simultaneous reverse transcription of target miRNAs and control according to the manufacturer's instructions.

\section{Real-time polymerase chain reaction amplification and analysis}

Real-time polymerase chain reaction (PCR) was performed using TaqMan microRNA assays specific to the corresponding mature sequence analyzed. Quantitative PCR (qPCR) steps were run in the StepOne real-time PCR system (Applied Biosystems) using TaqMan ${ }^{\odot}$ MicroRNA assays 20× (Applied Biosystems, USA, part No.4427975), TaqMan ${ }^{\odot}$ Endogenous Control Assay 20× (Applied Biosystems, USA, part No.4427975), and TaqMan Universal Master Mix II, no UNG $1 \times 1 \mathrm{~mL}$ (Applied Biosystems, USA, part No.4440043).

\section{Data analysis: comparative CT method $\left(\Delta \Delta \mathrm{C}_{\mathrm{T}}\right)$}

The miRNA expression level was measured using the $C_{T}$ method. The expression for each miRNA is represented by the difference between its $C_{T}$ value and the average $\mathrm{C}_{\mathrm{T}}$ value of reference genes, per sample, within a given sample set [19]. We used U6 snRNA as the reference gene. The relative expression (fold change) for each candidate miRNA within each group was then calculated using the equation $2^{-\Delta \Delta C T}$ [20]. The $\Delta C_{T}$ for each miRNA in each sample was calculated as follows: $\Delta \mathrm{C}_{\text {Tsample }}=\mathrm{C}_{\text {TtargetmiRNA }}-\mathrm{C}_{\mathrm{TU} \text { (6snRNA }}$. Then, $\Delta \Delta \mathrm{C}_{\mathrm{T}}$ was calculated as follows: $\Delta \Delta \mathrm{C}_{\mathrm{T}}=\left(\mathrm{C}_{\text {TmiRNA }}-\mathrm{C}_{\mathrm{TU} \text { 6snRNA }}\right)$ patients $-\left(\mathrm{C}_{\text {TmiRNA }}-\mathrm{C}_{\mathrm{TU} \text { 6snRNA }}\right)_{\text {controls }}$.

\section{Statistical analysis}

Statistical analysis was conducted using IBM SPSS ${ }^{\bullet}$ Statistics version 22 (IBM ${ }^{\bullet}$ Corp., Armonk, NY, USA). Numerical data were expressed as mean and standard deviation or median and range as appropriate. Qualitative data were expressed as frequency and percentage. Pearson's chi-square test or Fisher's exact test was used to determine the relationship between qualitative variables.

The receiver-operating characteristic (ROC) curve was used to evaluate the area under the curve (AUC) and its 95\% confidence interval and for predicting the cut-off values. The diagnostic accuracy of miRNAs was evaluated by calculating the sensitivity, specificity, positive predictive value (PPV), negative predictive value (NPV), and total accuracy. All tests were twotailed. A $P$ value $<0.05$ was considered as statistically significant.

\section{Results}

This study was conducted on 60 Egyptian patients with BD and 25 age- and sex-matched healthy subjects (control group).

In the patient group, there were $54(90 \%)$ males and 6 (10\%) females with a mean age of $36.3 \pm 8.3$ years (range $23-65$ years). The control group consisted of 20 (80\%) males and 5 (20\%) females with a mean age of $36.2 \pm$ 10.4 years (range 21-60 years). The demographic data of the patients and the control group are presented in Table 2.

\section{Clinical features of patients with BD}

All patients were subjected to clinical assessments. The age at onset of BD ranged from 11 to 45 years with a mean of $27.25 \pm 7.61$ years, and the disease duration ranged from 1 to 37 years with a mean of $9.05 \pm 7.56$ years. Table 3 shows the clinical characteristics of the patients with BD.

Statistical analysis revealed a significantly earlier onset of disease in patients with skin lesions (mean $24.7 \pm 7.3$ years) than in patients with non-skin lesions (mean 29.2 \pm 7.4 years $)(P=0.024)$. There was a trend toward an earlier onset of disease in patients with ocular lesions (mean $25.9 \pm 7.1$ years) than in patients with non-ocular lesions (mean $29.5 \pm 8.0$ years $)(P=0.074)$. The proportion of vascular and neurological manifestations was greater in the late-onset patient group, but not reaching any statistical significance.

\section{Results of BDCAF (activity score) and severity score in patients with $\mathrm{BD}$}

The BD activity in all patients was evaluated using the BDCAF. The results revealed eye involvement in $65 \%$ of patients, followed by CNS manifestations in $45 \%$ and joint arthralgia in $33.3 \%$ of patients. Table 4 shows the BDCAF results of patients with $\mathrm{BD}$. The disease severity score was also evaluated for the patients, which revealed mild severity in 4 patients $(6.7 \%)$, moderate severity in 9 patients $(15 \%)$, and high severity in 46 patients $(78.3 \%)$.

Table 2 Comparison between demographic features of BD patients and controls

\begin{tabular}{lllll}
\hline & & Patients (total no. =60) & Controls (total no. = 25) & $\boldsymbol{P}$ value \\
\hline Age (years) & & $36.3 \pm 8.3$ & $36.2 \pm 10.4$ & 0.954 \\
Sex no. (\%) & Males & $54(90 \%)$ & $19(76 \%)$ & 0.168 \\
& Females & $6(10 \%)$ & $6(24 \%)$ & \\
\hline
\end{tabular}


Table 3 Clinical characteristics of the studied BD patients

\begin{tabular}{lll}
\hline & No. of pt. (total no. =60) & $\%$ \\
\hline Oral ulcer & 59 & 98.3 \\
Genital ulcer & 54 & 90 \\
Ocular lesion & 37 & 61.6 \\
Skin lesion & 26 & 43.3 \\
$\quad$ Erythema nodosum & 7 (of total 26) & 26.9 \\
$\quad$ Folliculitis & 6 (of total 26) & 23 \\
$\quad$ Papule & 1 (of total 26) & 3.8 \\
$\quad$ Papulopustular lesions & 2 (of total 26) & 7.6 \\
$\quad$ Pustule & 1 (of total 26) & 3.8 \\
Arthritis & 8 & 13.3 \\
Arthralgia & 8 & 13.3 \\
Neurological manifestations & 10 & 16.6 \\
Vascular manifestations & 31 & 51.6 \\
\hline
\end{tabular}

\section{Association between BD severity score and clinical manifestations of patients with BD}

We compared the clinical manifestations between patients with mild/moderate $(n=13)$ and those with severe $(n=46) \mathrm{BD}$ and found no statistically significant differences, except pulmonary vascular involvement and BD severity score that demonstrated a significant association. We observed that $38.4 \%$ of patients with mild/ moderate BD and $10.6 \%$ of patients with severe BD manifested pulmonary vascular involvement $(P=0.017)$ and arterial vascular involvement that was almost but not quite significant $(P=0.068)$ (Table 5$)$.

Table 4 Results of BDCAF in the studied BD patients

\begin{tabular}{lll}
\hline & No. of pt. (total no. =60) & $\%$ \\
\hline Headache & 18 & 30 \\
Mouth ulceration & 18 & 30 \\
Genital ulceration & 8 & 13.3 \\
Erythema & 6 & 10 \\
Skin pustules & 15 & 25 \\
Joint arthalgia & 20 & 33.3 \\
Joint arthritis & 19 & 31.6 \\
Nausea/vomiting/ & 9 & 15 \\
abdominal pain & & 10 \\
Diarrhea \& bleeding & 6 & \\
per rectum & & 65 \\
Eye involvement & 39 & 45 \\
Nervous system involvement & 27 & 28.3 \\
Major vessel involvement & 17 & Range \\
& Mean \pm standard & \\
Total current activity from 2006 & $2.35 \pm 2.25$ & $0-9$ \\
\hline
\end{tabular}

Medical treatment of patients with BD

All the study patients were under medical treatment to control various disease manifestations and other comorbidities. The majority of them received corticosteroid therapy throughout the course of their disease illness. Almost all patients were treated with corticosteroid therapy in the form of either oral corticosteroid (prednisone) (93.3\%) or pulse methylprednisolone $(0.5-1 \mathrm{~g})(41.6 \%)$.

Almost all patients with $\mathrm{BD}$ received immunosuppressive therapy to control various active manifestations of the disease. Biologic disease-modifying antirheumatic drugs primarily in the form of TNF- $\alpha$ blockers were used in $28.3 \%$ of patients, which comprised intravenous infusion of the drug infliximab (Remicade). Anticoagulant therapy was applied in $58.3 \%$ of patients. A significant association was observed between the severity of BD and pulse IV cyclophosphamide therapy $(P=0.004)$.

MiRNA-146a and miR-155 expression in patients with BD and control subjects

Patients with BD showed a median 0.217 fold change in miR-146a expression levels (range 0.001-7.831), whereas this value was 1.658 (range 0.099-6.361) in the control group, indicating a statistically significantly lower expression of miR-146a in the patients than in control subjects $(P<0.001)$.

Regarding miR-155 expression, patients with BD showed a median 0.996 fold change (range 0.03711.791), whereas the control subjects showed a median 1.133 fold change (range 0.011-10.265), with no statistically significant difference being found between both groups $(P=0.736)$ (Table 6$)$.

\section{Correlation analysis between miR-146a and miR-155} expression in patients with BD and between miR-146a/ miR-155, demographic data, and duration of BD

In patients with $\mathrm{BD}$, a statistically significant positive correlation was found between miR-146a and miR-155 expression $(P<0.001, r=0.780)$ (Fig. 1). However, no statistically significant correlation was found between miR-146a/miR-155 expression and the age of patients with $\mathrm{BD}$, age at onset, and disease duration (Table 7 ).

\section{Association between miR-146a expression and clinical manifestations and laboratory parameters in patients with BD}

There was no significant association between miR-146a expression and the different clinical manifestations of patients with $\mathrm{BD}$, including ocular versus non-ocular lesions, vascular versus nonvascular manifestations, and skin versus non-skin lesions (all $P=1.000$ ) and neuro$\mathrm{BD}$ versus non-neuro-BD $(P=0.793)$ (Table 8$)$. There was also no significant association between miR-146a expression and different laboratory parameters. 
Table 5 Association of severity level of $B D$ with clinical picture of $B D$

\begin{tabular}{|c|c|c|c|c|}
\hline & & \multicolumn{2}{|l|}{ Severity } & \multirow{3}{*}{$\boldsymbol{P}$ value } \\
\hline & & \multirow{2}{*}{$\begin{array}{l}\text { Mild/moderate } \\
\% \text { (total no. }=13 \text { ) }\end{array}$} & \multirow{2}{*}{$\begin{array}{l}\text { Severe } \\
\%(\text { total no. }=46)\end{array}$} & \\
\hline & & & & \\
\hline \multicolumn{2}{|l|}{ Mouth ulceration } & $22 \%($ no. $=13)$ & $78 \%($ no. $=46)$ & No p-value because of small number of cases within groups \\
\hline \multicolumn{2}{|l|}{ Genital ulceration } & $22.2 \%($ no. $=12$ ) & $77.8 \%($ no. $=42$ ) & 1 \\
\hline \multicolumn{2}{|l|}{ Skin pustules } & $26.9 \%($ no. $=7)$ & $73.1 \%($ no. $=19)$ & 0.387 \\
\hline \multicolumn{2}{|l|}{ Joint arthalgia } & $25 \%($ no. $=2)$ & $75 \%($ no. $=6)$ & 0.806 \\
\hline \multicolumn{2}{|l|}{ Joint arthritis } & $25 \%($ no. $=2)$ & $75 \%($ no. $=6)$ & 0.806 \\
\hline \multicolumn{2}{|l|}{ Eye involvement } & $18.9 \%($ no. $=7)$ & $81.1 \%($ no. $=30)$ & 0.512 \\
\hline \multicolumn{2}{|c|}{ Nervous sys involvement } & $20 \%($ no. $=2)$ & $80 \%($ no. $=8)$ & 0.889 \\
\hline \multirow[t]{3}{*}{ Vascular involvement } & Venous & $30.8 \%($ no. $=8)$ & $69.2 \%($ no. $=18)$ & 0.134 \\
\hline & Arterial & $0 \%($ no. $=0)$ & $100 \%($ no. $=10)$ & 0.068 \\
\hline & Pulmonary & $38.4 \%($ no. $=5)$ & $10.6 \%($ no. $=5)$ & 0.017 \\
\hline
\end{tabular}

\section{ROC analysis}

ROC curve analysis was performed to analyze the expression of miR-146a as a potential biomarker for diagnosing BD. The expression level of miR-146a at a cut-off value of 0.650 demonstrated a sensitivity of $80 \%$, a specificity of $64 \%$, a PPV of $84.2 \%$, an NPV of $57.1 \%$, and an accuracy of $75.3 \%$ with an AUC of 0.786 (95\% confidence interval 0.686-0.887) (Fig. 2).

\section{Association between the expression of miR-146a and miR-155 and the BD severity score and BDCAF}

No statistically significant difference was observed between the expression of miR-146a and miR-155 and the BD severity level. There was also no significant correlation between BDCAF and both the miRNAs (Table 9).

\section{Discussion}

The pathogenic process of autoimmune disorders, especially those affecting multi-system organs in the body, is always multifactorial and complicated. The immune dysfunctions may be caused due to several genetic or environmental factors that may intersect in the causation of the disease [21].

In the present study, the expression levels of two miRNAs in patients with BD were investigated in an attempt to disclose the possible regulatory effects exerted by these miRNAs on the molecular pathways affecting the disease pathogenesis and to understand their immunologic effects on different clinical presentations, disease activity, and severity of symptoms. Therefore, the

Table 6 Comparison between BD patients and control regarding level of miR146a and miR155expression

\begin{tabular}{llll}
\hline Variable & Behcet $(\boldsymbol{n}=60)$ & Control $(\boldsymbol{n}=25)$ & $\boldsymbol{P}$ value \\
\hline miR-146a & $0.217(0.001-7.831)$ & $1.658(0.099-6.361)$ & $<\mathbf{0 . 0 0 1}$ \\
miR-155 & $0.996(0.037-11.791)$ & $1.133(0.011-10.265)$ & 0.736 \\
\hline
\end{tabular}

expression levels of two selected miRNAs (miR-146a and miR-155) were examined using quantitative realtime qRT-PCR in the serum of 60 adult patients with $\mathrm{BD}$, diagnosed according to the International Study Group for Behçet's disease criteria, and 25 healthy controls. We observed that miR-146a expression was significantly lower in patients with BD than in the control group, whereas miR-155 expression showed no statistically significant difference between both groups. There was also no significant association between the expression of miR-146a or miR-155 and the BDCAF.

These results collectively suggest that a downregulated miR-146a expression is involved in the pathogenesis of $\mathrm{BD}$.

MiRNAs are known to be involved in several physiological and pathological processes by targeting multiple functionally related proteins or key regulators of the immune system or the inflammatory response and by modulating the differentiation and response of various immune cells [22-25].

MiR-146a could exert its role in BD pathogenesis possibly through its anti-inflammatory role via a negative feedback regulation loop involving the downregulation of NF- $\mathrm{kB}$ to regulate excessive inflammation in response to proinflammatory stimuli, and its altered expression has been related to pathological conditions of the immune system [26].

Results of the present study are in general different from earlier reports. Kolahi et al. [27] showed that there was no significant difference in the miR-146a expression level between patients with BD and controls. Hou et al. [8] also reported that miR-146a did not confer susceptibility to $\mathrm{BD}$. In contrast to our results, Ibrahim et al. [28] found that miRNA-146a expression was significantly higher in patients with BD than in controls.

Interestingly, previous research showed that overexpression of miR-155 could significantly inhibit intracellular IL- 


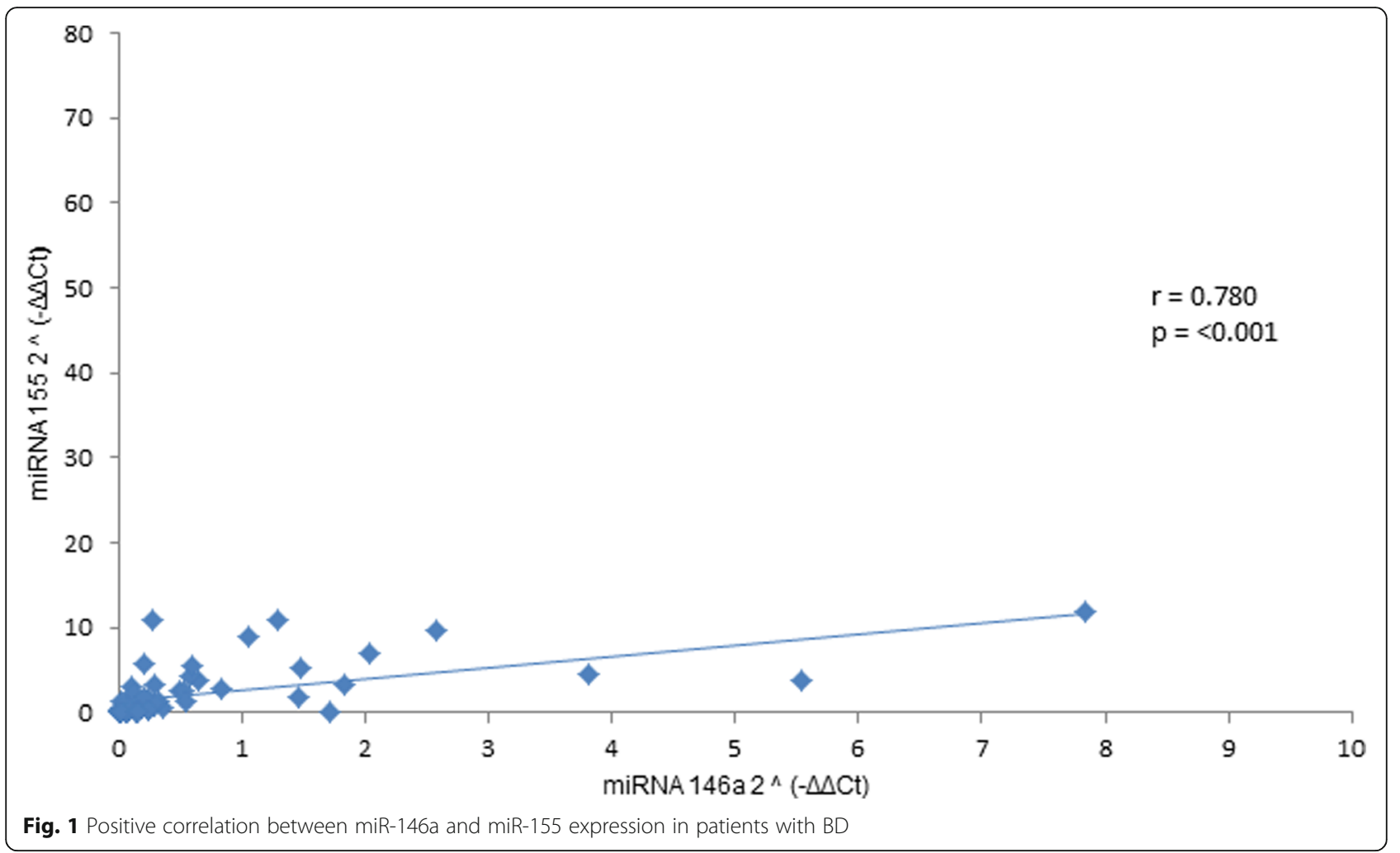

17 production by CD4T cells, whereas a downregulated miR-155 expression promoted intracellular IL-17 production by CD4-T cells. Collectively, these results suggest that a decreased expression of miR-155 can lead to the production of certain proinflammatory cytokines and the downregulated expression of IL-10, an important antiinflammatory cytokine, thus contributing to BD development [22]. Therefore, in the present study, we attempted to delineate the role of miR-155 in BD pathogenesis by measuring its expression level in patients with $\mathrm{BD}$, but we did not find any statistically significant difference in its expression level between the patients and control subjects. This result is in contrast to that reported by Kolahi et al. [27], who found that miRNA-155 expression was significantly increased in patients with compared to that in healthy controls. This difference could be attributed to the fact that all the examined patients were either under corticosteroid treatment or under other immunosuppressive therapy.

Table 7 Correlation between miR146a/miR155 and the age of $\mathrm{BD}$ patients, age at disease onset, and duration of $\mathrm{BD}$

\begin{tabular}{llllll}
\hline & \multicolumn{2}{l}{ miR146a } & & miR155 & \\
\cline { 2 - 3 } & $\boldsymbol{r}$ & $\boldsymbol{P}$ value & & $\boldsymbol{r}$ & $\boldsymbol{P}$ value \\
\hline Age & -0.049 & 0.707 & & 0.083 & 0.528 \\
Age at disease onset & 0.139 & 0.289 & & 0.229 & 0.079 \\
Duration of disease & -0.227 & 0.081 & & -0.192 & 0.142 \\
\hline
\end{tabular}

We also found no statistically significant correlation between miR-146a or miR-155 expression and the gender of patients with BD. Similarly, Ibrahim et al. [28] also reported the lack of a statistically significant difference between males and females in terms of miRNA146a expression.

In the present study, no significant correlation was detected between BDCAF and the examined miRNAs. Consistent with our result, Kolahi et al. [27] reported no significant association between the expressions of miR155 and miR-146a and BD activity. There was also no significant association between miR-146a expression and the different clinical presentations of patients with $\mathrm{BD}$.

Table 8 Association of age at disease onset with different clinical manifestations of $\mathrm{BD}$

\begin{tabular}{lll}
\hline & \multicolumn{2}{l}{ Age at disease onset } \\
\cline { 2 - 3 } Clinical manifestations of BD & Mean \pm SD & $\boldsymbol{P}$ value \\
\hline Occular lesions & $25.9 \pm 7.1$ & $\mathbf{0 . 0 7 4}$ \\
Non-occular lesions & $29.5 \pm 8.0$ & \\
Vascular manif. & $27.5 \pm 7.8$ & 0.757 \\
Non-vascular manif. & $26.9 \pm 7.5$ & \\
Skin lesions & $24.7 \pm 7.3$ & $\mathbf{0 . 0 2 4}$ \\
Non-skin lesions & $29.2 \pm 7.4$ & \\
Neurobehcet & $29.8 \pm 7.3$ & 0.241 \\
Non-neurobehcet & $26.7 \pm 7.6$ & \\
\hline
\end{tabular}




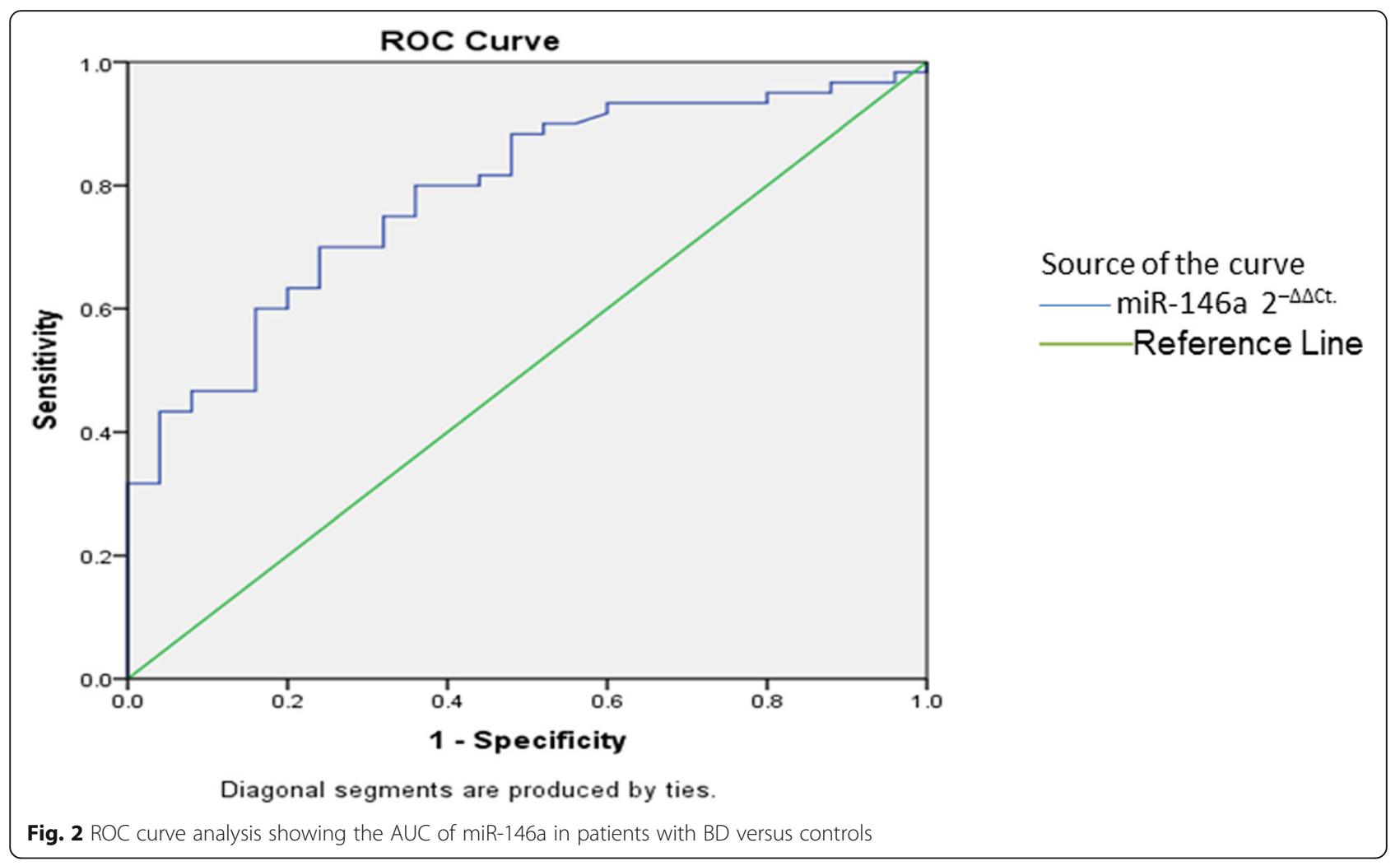

However, in contrast to our results, Ibrahim et al. [28] reported a significant association between miRNA-146a expression and both eye activity and vascular activity in patients with BD. In addition, Hassouna et al. [29] reported that miRNA-155 expression was significantly decreased with the increase in the BD activity assessed by BDCAF. Furthermore, Tili et al. [30] found that miRNA-155 expression was increased with BD activity.

A statistically significant positive correlation was found between miR-146a and miR-155 expression in patients with BD.

Table 9 Correlation between the expression of miR-146a and miR-155 with BD severity level and activity form BDCAF

\begin{tabular}{lllll}
\hline & & \multicolumn{2}{l}{ Severity } & P value \\
\cline { 3 - 4 } miR-146a & Mean \pm SD & $0.458 \pm 0.485$ & $0.732 \pm 1.502$ & 0.374 (NS) \\
& Range & $0.056-1.473$ & $0.001-7.831$ & \\
miR-155 & Mean \pm SD & $2.806 \pm 3.491$ & $2.143 \pm 2.872$ & 0.286 (NS) \\
& Range & $0.179-11.001$ & $0.037-11.791$ & \\
& & Total current activity form 2006 & \\
miR-146a & $r$ & -0.026 & & \\
& $P$ & 0.843 (NS) & & \\
miR-155 & $r$ & 0.084 & & \\
& $P$ & 0.521 (NS) & & \\
\hline
\end{tabular}

The findings of some previous studies are either consistent or inconsistent with our study results, which may be due to different ethnicities, different clinical manifestations, and disease severity and activity and also due to environmental factors among patients in the different studies.

The different miRNA expressions may be related to the effect and duration of the immunosuppressive treatment; however, there is a limited number of previous comparative studies.

It is important to mention that there are some limitations in our study. All our patients were on regular treatment with immunosuppressive agents. Our study results demonstrated a decreased expression of miR$146 \mathrm{a}$ in patients with $\mathrm{BD}$, and no difference was found in the miR-155 expression level compared with control subjects. However, it is not clear whether these levels were affected by therapy as it was not possible to include treatment-naive patients with $\mathrm{BD}$. It is also not clear whether the effect of miR-155 expression may be exerted by other immune cells or cytokine levels or a combination of both.

\section{Conclusion}

The present study showed that miR-146a expression was downregulated in patients with $\mathrm{BD}$ in comparison with healthy controls. The downregulated miR-146a expression could be involved in the pathogenesis of $\mathrm{BD}$ 
through a loss of control concerning the production of proinflammatory cytokines by DCs and CD4-T cells. Therefore, miR-146a may be used as a potential biomarker for the diagnosis of $\mathrm{BD}$ and as a therapeutic target in the future. However, our study did not reveal any significant difference in the miR-155 expression level between patients with BD and control subjects. There was also no association between BD activity and miR-146a and miR-155 expression levels.

\section{Future directions}

A study with a larger sample size is recommended to confirm the utility of miR-146a in the diagnosis and follow-up of patients with BD and to evaluate its potential role as a therapeutic target. Further studies are also required to focus on miR expression and its effect on BD activity and severity level, to link the expression of more miRs to pathological conditions of the immune system, and to correlate miR expression with immunosuppressive medications.

\section{Abbreviations}

AUC: Area under the curve; BD: Behçet's disease; BDCAF: Behçet's Disease Current Activity Form; ICBD: International Criteria of Behçet's Disease; miRNAs: MicroRNAs; NPV: Negative predictive value; PPV: Positive predictive value; qRT-PCR: Quantitative reverse transcription real-time polymerase chain reaction; ROC: Receiver-operating characteristic; Th17: T-helper 17; Th22: Thelper 22; Treg: $T$ regulatory lymphocytes

\section{Acknowledgements}

None.

\section{Authors' contributions}

E E has made a major role in interpretation of data and writing the manuscript. G R analyzed and interpreted the patient data regarding the rheumatological disease and the activity. N A has made substantial contributions to the conception and design of the work. E A performed the molecular acquisition, analysis, and interpretation of data. A W was a major contributor in writing and publishing the manuscript. The authors read and approved the final manuscript

\section{Funding}

This work was self-funded by the authors.

\section{Availability of data and materials}

The datasets used and/or analyzed during the current study are available from the corresponding author on reasonable request.

\section{Ethics approval and consent to participate}

All measures performed were in accordance with the ethical standards of Kasr-Alainy Cairo University Hospitals and with the 1964 Helsinki Declaration ethical standards. Research ethics committee of Cairo University Hospitals approved the study protocol No: I.230317. Written informed consent was obtained directly from each subject before data collection and after explanation of the study objectives

\section{Consent for publication}

The authors agree for the publication of the article.

\section{Competing interests}

The authors declare that they have no competing interests.

\section{Author details}

'Clinical and Chemical Pathology Department, Faculty of Medicine, Cairo University, Cairo, Egypt. ${ }^{2}$ Rheumatology and Rehabilitation Departement, Faculty of Medicine, Cairo University, Cairo, Egypt.

Received: 27 May 2020 Accepted: 17 July 2020

Published online: 23 October 2020

\section{References}

1. Alpsoy E (2016 Jun) Behcet's disease: a comprehensive review with a focus on epidemiology, etiology and clinical features, and management of mucocutaneous lesions. The journal of dermatology. 43(6):620-632

2. Nair JR, Moots RJ (2017 Feb) Behcet's disease. Clinical medicine. 17(1):71

3. El Menyawi MM, Raslan HM, Edrees A (2009 Apr) Clinical features of Behcet's disease in Egypt. Rheumatology international. 29(6):641-646

4. Dabbagh F, Haghighi AB, Ghasemi Y (2014 Apr) Behcet's disease: from heat shock proteins to infections. Asian biomedicine. 8(2):139-155

5. Alkhachroum AM, Saeed S, Kaur J, Shams T, De Georgia MA (2016 Mar) A case of neuro-Behcet's disease presenting with central neurogenic hyperventilation. The American journal of case reports. 17:154

6. Hatemi G, Seyahi E, Fresko I, Talarico R, Hamuryudan V (2015 Dec) Behçet's syndrome: a critical digest of the 2014-2015 literature. Clinical and experimental rheumatology. 33(6 Suppl 94):S3-S14

7. Davatchi F, Assaad-Khalil S, Calamia KT, Crook JE, Sadeghi-Abdollahi B, Schirmer M, Tzellos T, Zouboulis CC, Akhlagi M, Al-Dalaan A, Alekberova ZS (2014 Mar) International Team for the Revision of the International Criteria for Behçet's Disease (ITR-ICBD). The International Criteria for Behçet's Disease (ICBD): a collaborative study of 27 countries on the sensitivity and specificity of the new criteria. Journal of the European academy of dermatology and venereology. 28(3):338-347

8. Hou S, Ye Z, Liao D, Bai L, Liu Y, Zhang J, Kijlstra A, Yang P (2016 Jan) miR23a, miR-146a and miR-301a confer predisposition to Vogt-Koyanagi-Harada syndrome but not to Behcet's disease. Scientific reports. 6(1):1-9

9. Guarnieri DJ, DiLeone RJ (2008 Jan) MicroRNAs: a new class of gene regulators. Annals of medicine. 40(3):197-208

10. Bartel DP (2009 Jan) MicroRNAs: target recognition and regulatory functions. Cell. 136(2):215-233

11. Paul P, Chakraborty A, Sarkar D, Langthasa M, Rahman M, Bari M, Singha RS, Malakar AK, Chakraborty S (2018 Mar) Interplay between miRNAs and human diseases. Journal of cellular physiology. 233(3):2007-2018

12. Testa U, Pelosi E, Castelli G, Labbaye C. miR-146 and miR-155: two key modulators of immune response and tumor development. Non-coding RNA. 2017 Sep;3(3):22.

13. Chen HF, Hu TT, Zheng XY, Li MQ, Luo MH, Yao YX, Chen Q, Yu SY (2013 Jun) Association between miR-146a rs2910164 polymorphism and autoimmune diseases susceptibility: a meta-analysis. Gene. 521(2):259-264

14. Boldin MP, Taganov KD, Rao DS, Yang L, Zhao JL, Kalwani M, Garcia-Flores Y, Luong M, Devrekanli A, Xu J, Sun G (2011 Jun) miR-146a is a significant brake on autoimmunity, myeloproliferation, and cancer in mice. The journal of experimental medicine. 208(6):1189-1201

15. Muhammad JS, Ishaq M, Ahmed K (2019 Feb) Genetics and epigenetics mechanism in the pathogenesis of Behçet's disease. Current rheumatology reviews. 15(1):7-13

16. Puccetti A, Pelosi A, Fiore PF, Patuzzo G, Lunardi C, Dolcino M (2018 Oct) MicroRNA expression profiling in Behçet's disease. Journal of immunology research. 2018

17. Gul FC, Nazik H, Cicek D, Demir B (2017 May) Activity criteria in Behçet's disease. Behcet's Disease. 97

18. Krause I, Uziel Y, Guedj D, Mukamel M, Harel L, Molad Y, Weinberger A (1999 May) Childhood Behçet's disease: clinical features and comparison with adult-onset disease. Rheumatology. 38(5):457-462

19. Mestdagh $P$, Van Vlierberghe $P$, De Weer A, Muth D, Westermann F, Speleman F, Vandesompele J (2009 Jun) A novel and universal method for microRNA RT-qPCR data normalization. Genome biology. 10(6):R64

20. Schmittgen TD, Livak KJ (2008 Jun) Analyzing real-time PCR data by the comparative C T method. Nature protocols. 3(6):1101

21. Anaya JM, Ramirez-Santana C, Alzate MA, Molano-Gonzalez N, RojasVillarraga A (2016 Apr) The autoimmune ecology. Frontiers in immunology. 7:139

22. Ceppi M, Pereira PM, Dunand-Sauthier I, Barras E, Reith W, Santos MA, Pierre P (2009 Feb) MicroRNA-155 modulates the interleukin-1 signaling pathway 
in activated human monocyte-derived dendritic cells. Proceedings of the national academy of sciences. 106(8):2735-2740

23. Xiao C, Rajewsky K (2009 Jan) MicroRNA control in the immune system: basic principles. Cell. 136(1):26-36

24. Lin YC, Kuo MW, Yu J, Kuo HH, Lin RJ, Lo WL, Yu AL (2008 Oct) c-Myb is an evolutionary conserved miR-150 target and miR-150/c-Myb interaction is important for embryonic development. Molecular biology and evolution. 25(10):2189-2198

25. Xiao C, Calado DP, Galler G, Thai TH, Patterson HC, Wang J, Rajewsky N, Bender TP, Rajewsky K (2007 Oct) MiR-150 controls B cell differentiation by targeting the transcription factor c-Myb. Cell. 131(1):146-159

26. Tahamtan A, Teymoori-Rad M, Nakstad B, Salimi V (2018 Jun) Antiinflammatory microRNAs and their potential for inflammatory diseases treatment. Frontiers in immunology. 9:1377

27. Kolahi S, Farajzadeh MJ, Alipour S, Abhari A, Farhadi J, Bahavarnia N, Mahdavi AM, Khabbazi A, Sakhinia E (2018 Dec) Determination of mir-155 and mir-146a expression rates and its association with expression level of TNF- $\alpha$ and CTLA4 genes in patients with Behcet's disease. Immunology letters. 204:55-59

28. Ibrahim W, Sakr BR, Obaya E, Ghonem H (2019 Feb) MicroRNA-146a expression and microRNA-146a rs2910164 polymorphism in Behcet's disease patients. Clinical rheumatology. 38(2):397-402

29. Hassouna SS, Tayel MY, ElKaffash DM, Abdelhady AM, Elsayed EH (2018) MicroRNA155 expression in relation to BDCAF scored Behçet's disease in an Egyptian patients' sample. The open rheumatology journal. 12:115

30. Tili E, Michaille JJ, Cimino A, Costinean S, Dumitru CD, Adair B, Fabbri M, Alder H, Liu CG, Calin GA, Croce CM (2007 Oct) Modulation of miR-155 and miR-125b levels following lipopolysaccharide/TNF-a stimulation and their possible roles in regulating the response to endotoxin shock. The journal of immunology. 179(8):5082-5089

\section{Publisher's Note}

Springer Nature remains neutral with regard to jurisdictional claims in published maps and institutional affiliations.

\section{Submit your manuscript to a SpringerOpen ${ }^{\circ}$ journal and benefit from:}

- Convenient online submission

- Rigorous peer review

- Open access: articles freely available online

- High visibility within the field

- Retaining the copyright to your article

Submit your next manuscript at $\boldsymbol{\nabla}$ springeropen.com 